\title{
Índice de Madurez Digital para Empresas Hoteleras Familiares de Veracruz, México
}

\section{Digital Maturity Index for Family Hotel Companies in Veracruz, México}

\author{
Beatriz Eugenia Salas Parada ${ }^{1}$ \\ Universidad Veracruzana \\ https://orcid.org/0000-0001-9126-8253 \\ Guadalupe Juárez Gómez ${ }^{2}$ \\ Universidad Veracruzana \\ https://orcid.org/0000-0002-4710-0485 \\ Tomás Cuauhtémoc Carmona Cuervo ${ }^{3}$ \\ Universidad Veracruzana \\ https://orcid.org/0000-0002-5405-988X
}

\section{Cita Recomendada}

Recibido: 25-09-2020

Aceptado: 21-12-2020

Salas, B., Juárez, G. \& Carmona, T. (2020). Indice de madurez digital para empresas hoteleras familiares de Veracruz, México. Hamut'ay, 7 (3), 11-29.

http://dx.doi.org/10.21503/hamu.v7i3.2187

\section{Resumen}

El artículo muestra la definición y medición del índice de madurez digital en hoteles ubicados en el centro histórico de la ciudad de Veracruz, se resalta la importancia de este como parámetro para iniciar la transformación digital; es fundamental que las empresas tengan una hoja de ruta ante tareas impostergables como la Transformación Digital. Medir la madurez digital es un primer paso que refleja en sus diversas dimensiones, dónde se encuentran situadas las empresas seleccionadas, para con ello no retrasar más la decisión que tanta reticencia ha generado: usar tecnologías e incrementar su productividad y con ello su competitividad. La población de estudio se integró por Pymes hoteleras que ofrecen servicio de alojamiento. Se utilizó el cuestionario para la recolección de datos, permitiendo estudiar las dimensiones: Aprendizaje Organizacional, Cambio Organizacional, Recursos Humanos y Tecnología. Y se tuvo como objetivo el evaluar el índice de madurez digital. Los resultados muestran que las empresas se encuentran en niveles incipientes de uso de tecnologías, por lo que el nivel de madurez digital es prácticamente inexistente. Las 4 dimensiones estudiadas con las cuáles podían alcanzar 99 puntos en total, se encontraron con los siguientes puntajes promedios: Cambio Organizacional con 4.33 de 22 puntos totales, Tecnología 14.16 de 48 puntos totales, Aprendizaje Organizacional 8.26 de 19 puntos totales, Recursos Humanos 2.66 de 10 puntos totales.

1. Docente de la Universidad Veracruzana-México. Dedicada a la línea de investigación: Aplicación de las TIC en la solución de problemas de productividad en las organizaciones.bsalas@uv.mx.

2. Docente de la Universidad Veracruzana-México. Dedicada a la línea de investigación: Aplicación de las TIC en la solución de problemas de productividad en las organizaciones.gujuarez@uv.mx.

3. Docente de la Universidad Veracruzana-México. Dedicado a la línea de investigación: Aplicación de las TIC en la solución de problemas de productividad en las organizaciones. tocarmona@uv.mx. 
Cabe mencionar que esta medición dará pauta a una siguiente etapa que es el diseño de un Modelo de Transformación Digital para PyMES hoteleras familiares de la ciudad de Veracruz, México.

Palabras Clave: Transformación digital, madurez digital, índice de madurez digital, empresas hoteleras.

\begin{abstract}
The article shows the definition and measurement of the digital maturity index in hotels located in the historic center of the city of Veracruz, highlighting the importance of this as a parameter to initiate digital transformation; it is essential that companies have a roadmap to urgent tasks such as Digital Transformation. Measuring digital maturity is a first step that reflects in its various dimensions, where the studied companies are, so as not to delay further the decision that has generated such reluctance: use technologies and increase their productivity and thus their competitiveness. The study population was integrated by hotel SMEs offering accommodation service. The questionnaire was used for data collection, allowing study of the dimensions: Organizational Learning, Organizational Change, Human Resources and Technology. And it was aimed at evaluating the digital maturity index. The results show that companies are at emerging levels of technology use, so the level of digital maturity is virtually non-existent. The 4 dimensions studied with which they could reach 99 points in total, were found with the following average scores: Organizational Change with 4.33 of 22 total points, Technology 14.16 of 48 total points, Organizational Learning 8.26 of 19 total points, Human Resources 2.66 of 10 total points.
\end{abstract}

It should be mentioned that this measurement will lead to a next stage which is the design of a Digital Transformation Model for family hotel SMEs in the city of Veracruz, Mexico.

Key words: Digital transformation, digital maturity, digital maturity index, hotel companies.

\section{Introducción}

La situación sanitaria por COVID-19, ha puesto en evidencia, lo que muchas empresas no habían querido decirse a sí mismas, "vamos tarde en el uso de tecnologías de información". En cada ámbito de la vida las tecnologías tomaron un rol preponderante durante el confinamiento al que prácticamente el mundo entero se ha visto obligado, no sólo convirtieron hogares en oficinas o escuelas, permitieron mantener contacto con amigos y familiares y dispararon el uso de tiendas en línea: prácticamente cualquier producto podía llegar a la puerta de las casas sin que se tuviera que salir de ese espacio seguro; esto inevitablemente deja ante las implicaciones que tiene no seguir la ruta tecnológica "dejar de ser vistos por clientes y por lo tanto accesibles".
Las diversas tecnologías digitales se han ido integrando paulatinamente en las organizaciones, aunque no siempre al ritmo o en la forma idónea; también sabemos que incorporando tecnología las grandes empresas se vuelven líderes y este liderazgo está relacionado con el tamaño del presupuesto asignado a esta tarea.

Desde el nacimiento de la primera computadora programable creada hacia finales de los años treinta y hasta mediados de los años noventa cuando el término Transformación digital toma auge, estábamos encaminándonos a este momento de la historia, en la cuál las tecnologías finalmente dejaron de ser sustentadoras de los procesos organizacionales, y se convertirían en propiciadoras de nuevos modelos de negocio; un parteaguas que deja atrás las diferencias entre industrias, giros o el tamaño de la organización 
colocándolas a todas con una misma necesidad; incorporar tecnología a través de un proceso estratégico, que no deja fuera a ningún departamento o algún integrante del ecosistema empresarial; ya que en realidad parte fundamental de una verdadera transformación es que esta impregne el ADN de la empresa; las nuevas generaciones a la par que el desarrollo imparable de las tecnologías, pugnarán porque esta reinvención se concrete en todos los ámbitos de la vida y de los negocios, se sabe que las tecnologías no son una moda, y al ritmo propicio de cada organización, éstas deben ser incorporadas o contemplar el escenario de su desaparición.

El presente trabajo tiene como objetivo general evaluar el índice de madurez digital en hoteles ubicados en el centro histórico de la ciudad de Veracruz, como objetivos específicos: i) definir las dimensiones organizacionales que intervienen en la transformación digital de las empresas seleccionadas; ii) determinar el nivel de cada una de las dimensiones estudiadas; iii) conocer la madurez digital de las empresas. Se abordan los conceptos de transformación digital, índice de madurez, empresas familiares y se concluye con la descripción del estado que guarda la madurez digital en las empresas muestra de este estudio; el cuál se obtiene a través de la definición del índice de madurez, así como de su medición.

\section{Transformación Digital}

La Transformación digital es, de acuerdo a Perez \& Mejía (2018):

el cambio asociado con la aplicación de tecnología digital en todos los aspectos de la sociedad humana y puede ser considerada como la tercera etapa en la adopción de las tecnologías digitales: la competencia digital, el uso digital, la transformación digital, con la capacidad de uso y de capacidad transformadora que brinda la alfabetización digital. La etapa de transformación significa que los usos digitales permiten inherentemente nuevos tipos de innovación y creatividad en un ámbito particular, más que solo mejorar y apoyar los métodos tradicionales. (p. 24)
Desde la perspectiva de los autores, se considera que la transformación digital debe construir las bases de nuevas oportunidades para el negocio, transformándolo a través de diversas fases y convirtiéndolo, a fuerza de innovación, en un nuevo negocio.

Tomé (2017) citada por Juca et al., (2019) afirma que desde el punto de vista del negocio la transformación digital se trata de transformar tres áreas clave dentro de la organización: Modelos de negocios, la experiencia de usuarios, y las operaciones (p. 310). Incorporar las tecnologías a la empresa no es un proceso sencillo, ni rápido, es un proceso que implica planeación y que acompañará a las empresas en su transformación permanente o hasta su desaparición. De acuerdo con Schallmo \& Williams (2017) transformarse digitalmente implica que el recurso humano realice innovación, es decir deben reconsiderar los procesos actuales y crear nuevos, también la forma de tomar decisiones queda implicada en esta nueva forma de actuar y operar. Para De Esteban (2017) citado por Álvarez et al., (2019) no hay camino de retorno en la transformación digital; y considera que el término digital en sí mismo dejará de ser un diferenciador hacia el año 2022; ya que como resultado de esta transformación "lo digital" será una característica intrínseca en los productos y servicios; digitalizar procesos y la transformación que ello implica dejará de ser una meta para integrarse como una característica nuclear.

\section{Madurez Digital}

De acuerdo con Lorenzo (2016) comprender que la experiencia del cliente es personal e intransferible denota los primeros atisbos de madurez digital en la organización, a partir de aquí, los tomadores de decisiones comprenden el binomio cliente-dispositivos digitales y el impacto que las tecnologías tienen cuando el consumidor toma decisiones de compra. Las metas y objetivos organizacionales empiezan a ser alineadas a las iniciativas de transformación digital. 
En general, Simpson \& Weiner (1989) afirman que el término "madurez" se refiere a un "estado de completa, perfecta o lista" e implica cierto progreso en el desarrollo de un sistema, citado por (Schumacher, Erol, \& Sihn, 2016). En consecuencia, los sistemas de maduración (por ejemplo, biológicos, organizativos o tecnológicos) aumentan sus capacidades a lo largo del tiempo con respecto al logro de algún estado futuro deseable. La madurez se puede capturar cualitativamente o cuantitativamente de manera discreta o continua (Kohlegger, Maier \& Thalmann 2009) citado por (Schumacher, Erol, \& Sihn, 2016).

Los autores entendemos la madurez digital como los diversos estadios que recorre la empresa en su proceso transformativo, de tal manera que acorde a su nivel de uso e impacto las organizaciones tienen un nivel alcanzado en el uso de la tecnología en los procesos organizacionales, lo cual delimita su capacidad de respuesta a los retos del entorno y sin lugar a duda determina su productividad y competitividad. Este punto de vista da por sentado que las empresas se verán sometidas a una evolución constante, similar a la de los humanos en las distintas etapas de sus vidas. Como todo proceso de maduración, la digital es individual, progresiva y única en sus formas y tiempos, pero indiscutiblemente se da en el largo plazo; de manera que se permita el desarrollo de nuevas capacidades y habilidades en los miembros de la organización.

Para conocer el grado de madurez digital en el que se encuentran las organizaciones, se realiza una medición a través de indicadores que nos permiten conocer cuantitativamente el grado de madurez o avance alcanzado en la transformación digital; obteniendo así una medición objetiva de las debilidades y fortalezas encontradas en la organización; es un instrumento sumamente útil para conocer las capacidades que cuenta la empresa y las que no; para continuar la ruta de la transformación digital.

En el índice de Madurez Digital (IMD) se combinan dos dimensiones diferentes: el liderazgo (con visión digital) que impulsa la transformación, y la capacidad digital, aquella que hace referencia a la innovación tecnológica que realiza la organización para mejorar la gestión operativa y una mejora en su competitividad (Westerman, Bonnet y McAfee, 2012, 2014) citados por (Cuenca, Matilla, \& Compte, 2020). Sin embargo, para Lorenzo (2016) la serie de nuevas capacidades que se irán desarrollando con los recursos de la organización en el camino de la transformación; contemplan una lista más variada de dimensiones organizacionales. "El desarrollo de estas capacidades debe ocurrir de manera integrada en todas las dimensiones de la organización: estrategia, personas y cultura, estructura y sistemas de gestión, procesos de negocio y, por supuesto, en la tecnología" (Lorenzo, 2016, p. 574). Al ser la Transformación Digital un proceso que implica a todas las áreas del negocio y que debe sostenerse desde la planeación estratégica: se convierte en un proceso multidimensional, en el cual es necesario que las capacidades digitales se desarrollen e integren en todas las dimensiones anteriormente señaladas (Lorenzo, 2016).

En este estudio, el índice de madurez digital es el medio a través del cuál conocemos el avance de la transformación digital o grado de madurez en las empresas muestra; se logra a través de una serie de indicadores (recabados con el instrumento de medición); dichos indicadores permitieron una visión individual y global de los integrantes de la muestra. Estos indicadores fueron englobados en 4 Dimensiones; permitiendo retratar a la empresa y delimitar la serie de capacidades que deben ser desarrolladas en cada dimensión organizacional; y no hay que olvidar que existe una perpsectiva única para comprender a las organizaciones; pero si consideramos que las dimensiones establecidas en el estudio permiten obtener la imagen completa de nuestra muestra; permitiendo concretar el objetivo buscado. Una vez que se ha establecido que la transformación digital se da en las organizaciones a lo largo y ancho de sus dimensiones (las cuáles pueden ser diversas) y de manera integral; corresponde definir las dimensiones que fueron determinadas para el es- 
tudio; las cuáles se consideran, tanto las citadas por (Cuenca, Matilla, \& Compte, 2020), como las contempladas por (Lorenzo, 2016).

Ver la tabla 1 a continuación.

\section{Tabla 1}

Dimensiones del estudio

\begin{tabular}{cl}
\hline Dimensión & \multicolumn{1}{c}{ Descripción } \\
\hline Aprendizaje & $\begin{array}{l}\text { Relativa a la Creación y } \\
\text { adquisición de conocimiento, } \\
\text { así como la consolidación de }\end{array}$ \\
& una cultura organizacional, \\
& todo lo relativo a la creación de \\
& identidad y el fortalecimiento \\
& de la misma, considerando el \\
& tipo de liderazgo.
\end{tabular}

\begin{tabular}{cl}
\hline Cambio & Relativa a la innovación: \\
Organizacional & pequeños o grandes pasos en \\
& un sentido de mejora continua, \\
& dejando lo conocido y \\
& aprendiendo nuevas maneras \\
& de hacer las cosas; hasta nuevas \\
& formas de hacer negocios.
\end{tabular}

\begin{tabular}{cl}
\hline Recursos & $\begin{array}{l}\text { Relativo al recurso más } \\
\text { importante de la organización } \\
\text { (el recurso humano), } \\
\text { capacitación, promoción, } \\
\text { recompensas, productividad. }\end{array}$ \\
\hline Tecnologías & $\begin{array}{l}\text { Uso, operación, desarrollo de } \\
\text { aplicaciones de software y } \\
\text { hardware, así como ventajas } \\
\text { encontradas en su uso. }\end{array}$ \\
\end{tabular}

Fuente: Elaboración propia (2020)

Se sabe que, aunque comparten la necesidad de: transformarse digitalmente, los recursos y capacidades de cada organización son diversos, es por ello que, al conformarse la muestra de este estudio por empresas familiares, haremos una descripción general de las características que acompañan a este tipo de organizaciones.

\section{Empresas Familiares}

Para Herrera, (2015), la empresa familiar es una organización muy compleja integrada por dos universos que, aunque son totalmente distintos, se vuelven interdependientes y comunes: La empresa y la familia. (p. 90)

Sin embargo, la sencillez de ambos conceptos no se transfiere a la complejidad que implica su conjunción; ya que definir a las empresas familiares es complejo debido a que el universo integrado por empresas familiares es vasto; incluyendo desde "la tiendita de la esquina" hasta una trasnacional que cotiza en bolsa.

De acuerdo con Rodriguez (2018), las empresas familiares nacen de conocimientos previos de sus fundadores en ciertos mercados; otras veces como resultado de la casualidad y conjunción de factores que conllevan su creación como puede ser la apertura de una franquicia; lo que es muy real es que una idea vendible puede empezar un negocio; posterior a su nacimiento y las diversas causas que pueda tener el siguiente reto de una empresa familiar es llevar su ciclo de vida más allá de la tercera generación. Para ello será necesario que crezca y madure para no correr la misma suerte de la mayoría de las empresas familiares que desaparecen antes de alcanzar este objetivo; y que por el contrario pueda convertirse en un caso de éxito. Las empresas familiares no tienen límite en su crecimiento, su límite está determinado por la visión y capacidad de quien la guía.

Por su parte las empresas familiares hoteleras son concebidas como aquellos establecimientos o negocios de hospedaje que ofrecen los servicios básicos o complementarios de alojamiento, en donde la toma de decisiones y control la ejerce el fundador o propietario apoyado en algunas ocasiones por alguien de la familia que también participan en la gestión.

En la tabla 2 se observa como está conformada la muestra con respecto al relevo generacional, lo 
cual puede dar una idea general de la solidez y/o riesgo que enfrentan.

\section{Tabla 2}

Relevo generacional de la muestra estudiada

\begin{tabular}{lc}
\hline $\begin{array}{c}\text { Relevo } \\
\text { Generacional }\end{array}$ & $\begin{array}{c}\text { Número de } \\
\text { empresas }\end{array}$ \\
\hline Primera generación & 5 \\
\hline Segunda generación & 5 \\
\hline Tercera & 3 \\
\hline $\begin{array}{l}\text { Cinco generaciones } \\
\text { o más }\end{array}$ & 2 \\
\hline
\end{tabular}

Fuente: Elaboración propia (2020)

\section{Materiales y Métodos}

\section{Participantes}

Los sujetos involucrados en este estudio fueron un grupo de PyMES hoteleras familiares, localizadas en el Centro Histórico de la Ciudad y Puerto de Veracruz con la particular característica de prestar servicios básicos de hospedaje. En la tabla 3 se presentan datos de la población y muestra de estudio. A través de la observación, se realizó un inventario cuantitativo, con una muestra de 15 de ellos, y de esa forma se identificó el número de procesos sustentados en tecnología, lo cual permite establecer las diferencias entre cada una de esas organizaciones.

\section{Tabla 3}

Descripción de la muestra de estudio.

\begin{tabular}{cccc}
\hline $\begin{array}{c}\text { Población } \\
\text { (Núm. De } \\
\text { Hoteles) }\end{array}$ & $\begin{array}{c}\text { Muestra } \\
\text { (Núm. De } \\
\text { Hoteles) }\end{array}$ & $\begin{array}{c}\text { Clasificación de } \\
\text { acuerdo con el } \\
\text { Núm. de Estrellas }\end{array}$ & $\begin{array}{c}\text { Promedio de } \\
\text { colaboradores }\end{array}$ \\
\hline 31 & 1 & $\star \star \star \star \star$ & 52 \\
& 3 & $\star \star \star \star$ & 23 \\
\cline { 2 - 4 } & 9 & $\star$ & 6 \\
\hline Total & 15 & & \\
\hline
\end{tabular}

Fuente: Elaboración propia en base a la investigación

(2020)

Para la selección de la muestra se utilizó el muestreo no probabilístico, pues se desconocía la accesibilidad del total de empresas integrantes de la población. Este muestreo por conveniencia no utiliza un muestreo estadístico, sino que es determinado por el acceso a la información de los hoteles. En esta muestra se busca evaluar el índice de madurez digital que poseen, como un paso previo a proponer un modelo de adopción tecnológica o transformación digital que les permita un desarrollo sostenido.

\section{Instrumento}

El instrumento que se utilizó para el desarrollo del estudio fue el cuestionario, levantándose la información relacionada con las variables implicadas en la investigación y que posteriormente fueron asociadas a las dimensiones del índice de transformación digital. Dicho cuestionario estuvo diseñado con 35 reactivos, divididos en cuatro secciones; la primera permitió obtener los datos generales de Pymes de hospedaje; la segunda 
sección recaba información como: métodos utilizados para definir la tarifa; porcentaje de ocupación y ventas anuales; estudios que han hecho para identificar el rumbo que toman los competidores; programas de capacitación, entre otros. Por su parte, la tercera sección implica la obtención de datos como: en qué áreas o departamentos se han efectuado cambios; si han aplicado algunas estrategias de negocios; qué procesos se encuentran automatizados o, en todo caso, si tienen pensado invertir en recursos tecnológicos en los próximos años.

La cuarta sección, comprende datos como: el uso y propósito de los servicios de internet; qué aplicaciones informáticas se utilizan en la empresa y cuáles son sus logros; cuestiones referentes a las actualizaciones de las plataformas con las que cuentan las empresas objeto de estudio; y si disponen de un departamento de informática dentro de la estructura organizacional de la empresa. El instrumento, fue sometido a la medición de la confiabilidad, que consistió en la aplicación del instrumento (en un solo momento) a un grupo de PyMEs hoteleras de organización familiar, dedicadas al servicio de hospedaje localizadas en el Centro Historico de la zona de estudio, esta medición de confiabilidad a traves del coeficiente de Alfa de Cronbach arrojó los siguientes resultados en la tabla 4.

\section{Tabla 4}

Alfa de Cronbach

\begin{tabular}{lc}
\multicolumn{1}{c}{ Items } & Coeficiente \\
\hline Alfa de Cronbach & 0.852 \\
en los items que & \\
utilizan escala de & \\
likert & \\
\hline Alfa de Cronbach & 0.957 \\
referentes a la & \\
actualización de & \\
las plataformas &
\end{tabular}

Fuente: Elaboración propia (2020)
A que esta parte del cuestionario es evaluada como muy buena, asimismo un coeficiente de 0.957 , indica que esta parte queda evaluada como excelente. Ver anexo 1

\section{Tipo y Diseño}

El presente estudio se llevó a cabo con un diseño no experimental cuantitativo, el cual, de acuerdo con Hernández, Fernández \& Baptista (2014) "las variables independientes ocurren y no es posible manipularlas, no se tiene control directo sobre dichas variables ni se puede influir sobre ellas, porque ya sucedieron, al igual que sus efectos" (p.152).

Los datos de este estudio fueron recabados en un único momento, in situ, a través de la respuesta al instrumento diseñado, permitiendo la observación de la realidad de los hoteles muestra para su posterior análisis. Este estudio no experimental cuantitativo de cohorte transeccional o transversal nos permitió recolectar datos en un solo momento para conocer el índice de madurez digital en las diversas dimensiones organizacionales implicadas en el instrumento; razón por la cuál es descriptivo; su alcance no require la formulación de hipótesis ya que no intenta pronosticar una cifra o un hecho sino únicamente conocer a través de su medición los elementos o dimensiones organizacionales que intervienen en la transformación digital.

\section{Procedimiento}

Para el desarrollo de la investigación se diseñó un plan de trabajo dividido en fases con la finalidad de planificar y sistematizar las actividades a realizar:

\section{Fase I. Reunión de trabajo}

Se solicitó una reunión con el presidente de la Asociación de Hoteles y Moteles de la Ciudad de Veracruz para presentar el objetivo del proyecto, dejar antecedentes y señalar el propósito académico, en dicha reunión se acordó que la asociación extendería los oficios correspondientes para 
la aplicación del instrumento y levantamiento de la información logrando con esto una gran apertura y apoyo por parte de los empresarios hoteleros.

Fase II. Trabajo de Campo

Asignación de los hoteles de organización familiar localizados en el área de estudio en cuestión a los colaboradores para la recolección de información. Se efectuaron antes, durante y después de la recolección de información reuniones para la coordinación, planificación, ejecución y seguimiento del trabajo de campo.

Fase III. Análisis de datos

Para la codificación de la información, primero se revisaron cada uno de los cuestionarios para verificar la correcta respuesta de los indicadores asignándoles una numeración a cada uno de ellos, la información fue vaciada en una hoja de cálculo, generando la base de datos con las variables de estudio y las respuestas de cada uno de los ítems de los cuestionarios aplicados. Posteriormente se agruparon los ítems por dimensión, permitiendo así realizar un cálculo y posterior evaluación del índice de madurez digital de las empresas estudiadas.

\section{Consentimiento informado}

Para el abordaje realizado a los integrantes de la muestra, se contó con el apoyo de la Asociación de hoteles y moteles de Veracruz, quién les informó que un grupo de docentes de la Universidad Veracruzana estaba efectuando un estudio encaminado a evaluar el índice de madurez digital de sus establecimientos de hospedaje con la finalidad de ofrecer posibles rutas de mejora para la citada transformación, derivado de los anterior se logró el apoyo y participación para responder las preguntas del instrumento; no sin antes garantizarles el resguardo de su identidad respetando, así su confidencialidad.

\section{Resultados}

Para el presente estudio se evaluó un índice de madurez digital que permite conocer con objeti- vidad en qué situación se encuentran los hoteles muestra. Hablar de transformación digital es hablar de un proceso continuo, que para su inicio es necesario conocer a cabalidad el estado que guarda la empresa con respecto al uso de tecnologías; es de vital importancia conocer el punto de partida, para con ello establecer los pasos que se requieren para su transformación.

Enseguida se exponen los resultados del estudio en las figuras correspondientes. La figura 1 presenta los resultados que determinan el índice de madurez digital en el sector de servicios de hospedaje de acuerdo con las 4 dimensiones para las 15 empresas hoteleras.

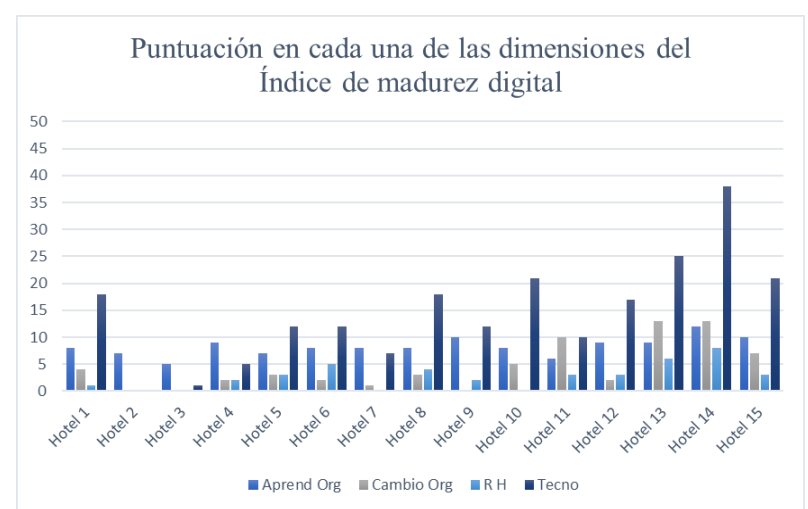

Figura 1. Puntuación en cada una de las dimensiones del índice de madurez digital. Fuente: Elaboración propia (2020).

La puntuación total alcanzable por las empresas muestra en este índice de madurez digital en cada una de sus dimensiones, así como el promedio de la muestra y los máximos y mínimos obtenidos se aprecian en la siguiente tabla 5 . 
Tabla 5

Puntuación de las dimensiones estudiadas.

\begin{tabular}{lccccc}
\hline \multicolumn{1}{c}{ Dimensión } & $\begin{array}{c}\text { Puntaje } \\
\text { máximo }\end{array}$ & Promedio & $\begin{array}{c}\text { Promedio en } \\
\text { Porcentaje }\end{array}$ & $\begin{array}{c}\text { Máximo } \\
\text { obtenido }\end{array}$ & $\begin{array}{c}\text { Mínimo } \\
\text { obtenido }\end{array}$ \\
\hline $\begin{array}{l}\text { Cambio } \\
\text { Organizacional }\end{array}$ & 22 Puntos & 4.33 Puntos & $19.68 \%$ & 13 Puntos & 0 Puntos \\
\hline Tecnología & 48 Puntos & 14.46 Puntos & $30.12 \%$ & 38 Puntos & 0 Puntos \\
\hline $\begin{array}{l}\text { Aprendizaje } \\
\text { Organizacional }\end{array}$ & 19 Puntos & 8.26 Puntos & $43.47 \%$ & 12 Puntos & 5 Puntos \\
\hline $\begin{array}{l}\text { Recursos } \\
\text { Humanos }\end{array}$ & 10 Puntos & 2.66 Puntos & $26.6 \%$ & 8 Puntos & 0 Puntos \\
\hline TOTAL & 99 Puntos & & & \\
\hline
\end{tabular}

Fuente: Elaboración propia (2020)

Como podemos apreciar en la Figura 1 y en la Tabla 5, hay mucho trabajo por hacer en lo referente a madurez digital, sólo uno de los hoteles se acerca al máximo obteniendo 38 de 48 puntos en la dimensión Tecnología, mientras que el promedio de la muestra en esta misma dimensión es de 14.46 puntos. Esto se menciona por ser el valor más representativo, sin embargo, también el promedio calculado en forma de porcentaje en cada una de las dimensiones tiene niveles que no superan el $50 \%$ del valor que podrían alcanzar. A continuación, se desglosa para cada Dimensión los resultados de su medición.

\section{Cambio Organizacional}

La dimensión Cambio organizacional cuyo resultado se muestra en la figura 2, está compuesta por cuatro indicadores: áreas a las cuáles se les asigna presupuesto en sus periodos correspondientes, información qué son examinados para llevar a cabo la toma de decisiones, estrategias qué considera en el corto plazo, y procesos que actualmente tiene automatizados.

El promedio alcanzado por la muestra para esta dimensión es 4.33 puntos de un máximo de 22 que podían obtener, el máximo logrado por dos hoteles es de 13 puntos; mientras que tres hoteles obtienen cero puntos, lo anterior demuestra que es inminente la necesidad de diseñar estrategias y proporcionar herramientas para enfrentar el Cambio Organizacional por parte de las Pymes que ofrecen servicio de hospedaje.

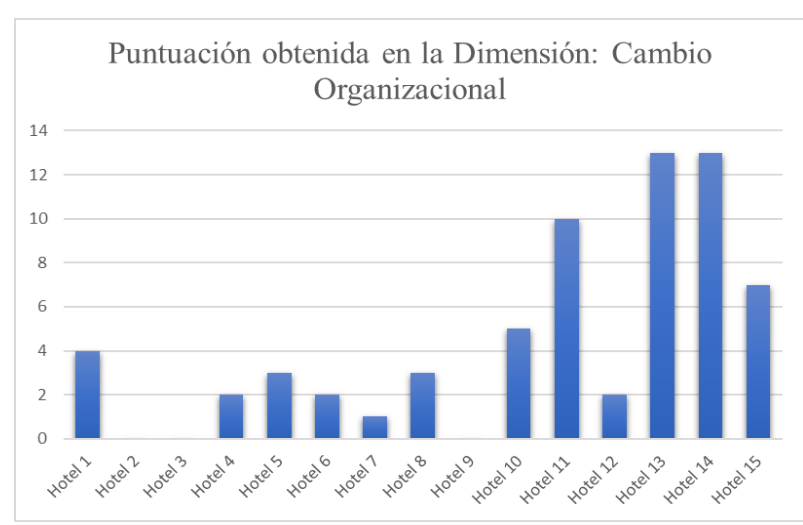

Figura 2. Puntuación obtenida Dimensión: Cambio Organizacional.

Fuente: Elaboración propia (2020).

\section{Tecnología}

La dimensión Tecnología, está integrada por diez indicadores: servicios de internet que usan, propósito de uso de los servicios de internet, aplicaciones informáticas con las que cuentan, impacto o logros de las aplicaciones informáticas, apoyo 
obtenido por las TIC para la toma de decisiones, Nivel de actualización de las TIC, elementos que son considerados en la planeación de TIC de la empresa, si cuentan con departamento de informática, cuántas personas hay en el departamento de informática, y por último actividades que son realizadas por el departamento mencionado. En la figura 3 se observa que el promedio de la muestra para esta dimensión es de 14.46 puntos de un máximo de 48 que podían obtener, el máximo conseguido por un hotel de la muestra es de 38 puntos mientras que otro de los hoteles se queda en cero puntos, indicadores que manifiestan que es urgente la incorporación de las tecnologías en el tipo de empresas del tejido empresarial investigado. La tecnología ha sido y es un factor determinante en la gestión de hospedaje porque provee valor agregado entre empresa y cliente siendo un medio directo de comunicación, difusión, venta, al mismo tiempo que satisface la exigencia de un mercado altamente competitivo.

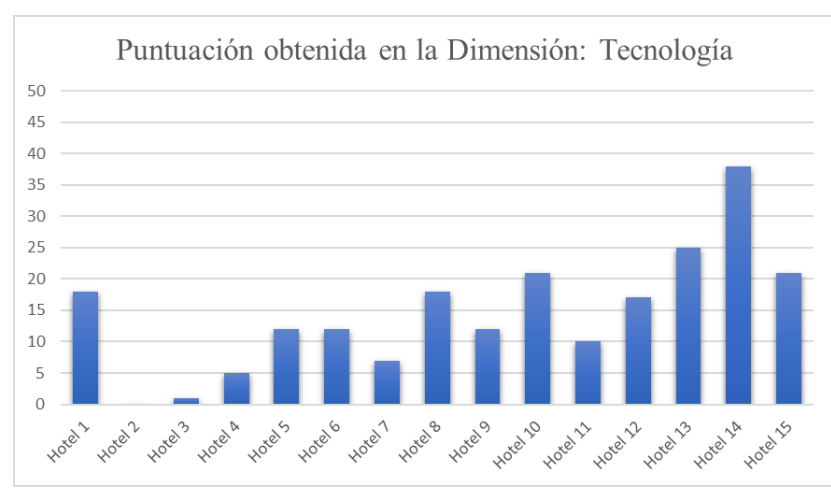

Figura 3. Puntuación obtenida en la Dimensión: Tecnología

Fuente: Elaboración propia (2020).

\section{Aprendizaje organizacional}

La dimensión Aprendizaje Organizacional, se compone de cuatro indicadores: método tarifario utilizado, ocupación anual promedio y la diversificación de su ocupación en los diversos mercados (regional, estatal, nacional e internacional). En la figura 4, se aprecia el promedio de la muestra para esta dimensión es 8.26 puntos de un máximo de 19 que podían obtener, el máximo obtenido por un hotel es de 12 puntos mientras que el mínimo conseguido por uno de los hoteles es de 5 puntos, aquí se observa que esta es la dimensión con mejor puntaje de las cuatro analizadas.

El aprendizaje organizacional se muestra como una elección de la transformación de conocimiento entre los miembros de la organización que buscan ser productivos, competitivos y eficientes, es decir; aprovechar al máximo las capacidades y oportunidades identificadas.

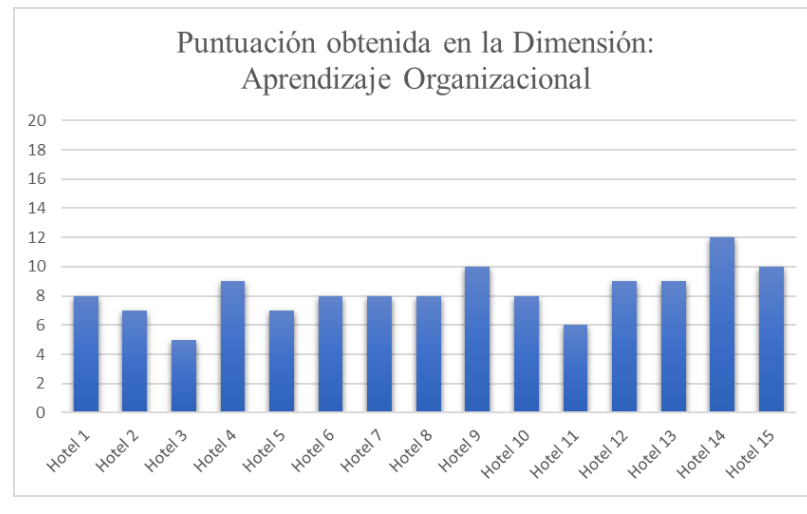

Figura 4. Puntuación obtenida en la Dimensión: Aprendizaje Organizacional. Fuente: Elaboración propia (2020).

\section{Recursos humanos}

La dimensión recursos humanos está diseñada por cuatro indicadores: programas de capacitación, periodicidad de la capacitación, programas de promoción y ascenso, y por último programa de recompensas.

El promedio para esta dimensión como lo representa la figura 5, es 2.66 puntos de un máximo de 10 que podían obtener, el máximo alcanzado por un hotel es de 8 puntos mientras que cuatro hoteles quedan en cero puntos, es importante el rol de los recursos humanos en los procesos de cambio digital ya que las personas son el centro de atención para impulsar o no aprobar la adopción a través de la creación de una cultura organizacional que sustente el cambio transformador instigado por las tecnologías. 


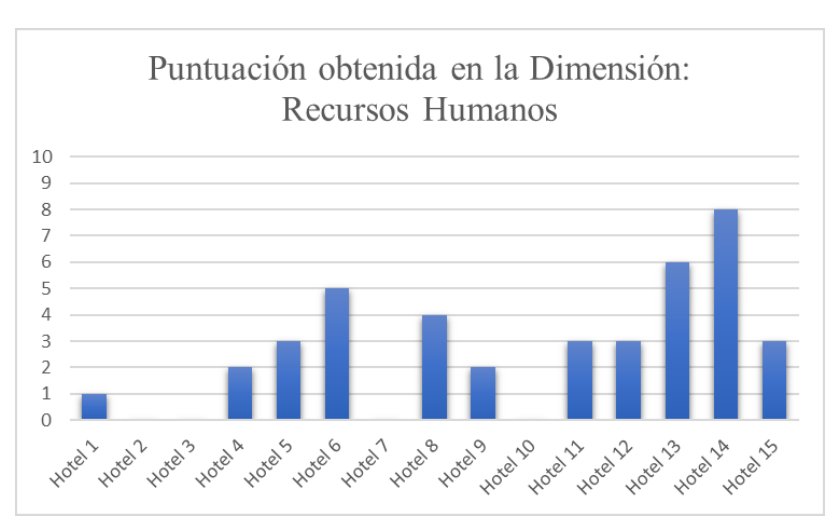

Figura 5. Puntuación obtenida en la Dimensión: Recursos Humanos.

Fuente: Elaboración propia (2020).

Las figuras previas nos desglosan cada una de las dimensiones, mientras que la siguiente figura (Figura 6), representa el resumen del índice de madurez digital alcanzado en cada uno de los hoteles de la muestra, el cuál integra las 4 dimensiones mencionadas.

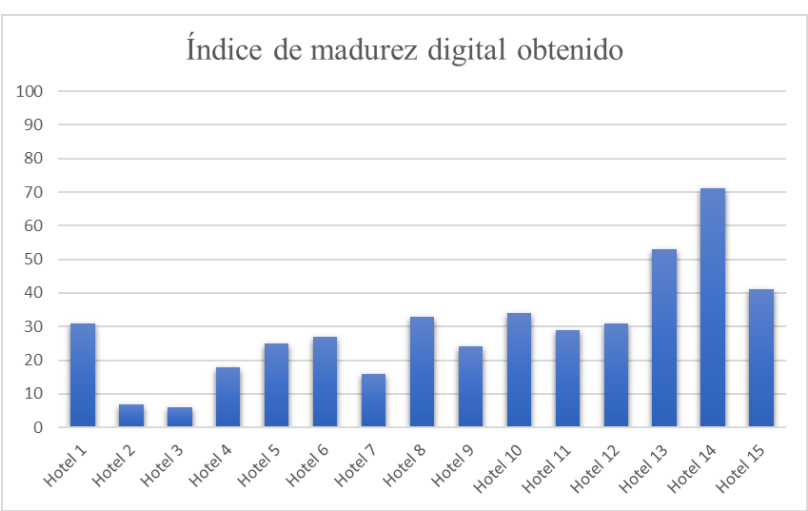

Figura 6. Índice de madurez obtenido. Fuente: Elaboración propia (2020).

Como puede apreciarse en la Figura 6. "Índice de madurez obtenido", en la muestra encontramos sólo dos empresas que alcanzaron más de 50 puntos, lo que puede leerse también como estar arriba del promedio, ya que el total posible es de 99 puntos. Estas dos empresas representan el $13.33 \%$ de Pymes; cuando se plantea de manera inversa y afirmamos que el $86.66 \%$ de las empresas no alcanzan este promedio; o que hay dos hoteles que no alcanzan el 10\% de los puntos posibles y que el promedio del índice de madurez digital está en $30 \%$ obtenemos una imagen preocupante del nivel de adopción con que cuentan.

\section{Conclusiones}

Las herramientas tecnológicas han estado disponibles para todas las organizaciones desde hace varios años, la resistencia de adoptarlas también, después de la pandemia, la adopción de la tecnología de información derribará esta resistencia o a la misma organización de manera inmediata. Las herramientas brindadas como apoyo por el sector público, el privado y diversas organizaciones, será fundamental para la recuperación económica en todos los niveles.

Este estudio sienta el precedente que permite la construcción de un modelo de transformación digital para las Pymes hoteleras.

Ante los constantes, inesperados e inciertos cambios económicos que se están presentando, es fundamental que el tejido empresarial del área de estudio considere oportuno el uso urgente de las herramientas tecnológicas; que sean el inicio de la transformación digital; herramientas aliadas como factor determinante para adaptarse a las exigencias de un mercado permanentemente competitivo. Estos cambios son un reto para las organizaciones, esto supone cambiar sus modelos de negocios, es decir; integrarse a una nueva cultura organizacional donde la estrategia principal sea transformarse digitalmente.

A través de este estudio se identificó que sólo dos empresas alcanzan más de 50 puntos de un total posible de 99 puntos posibles del Índice. Estas dos empresas representan el $13.33 \%$ de Pymes; De manera inversa esto denota que el $86.66 \%$ de las empresas no alcanzan este promedio; también se identifica que hay dos hoteles que no alcanzan el $10 \%$ de los puntos posibles y que el promedio del índice de madurez digital está en $30 \%$, estos datos nos arrojan una imagen preocupante del nivel de adopción con que cuentan.

Asimismo, los datos confirman la enorme necesidad y urgencia de incorporar o alinear estratégicamente la transformación digital en las empresas muestra, esto a través de un modelo guía que 
facilite la superación de esta crisis, y su posterior desarrollo competitivo.

Se concluye que la transformación digital en el sector estudiado tiene una gran oportunidad de desarrollo a través de estrategias que les permitan la inclusión digital y no perder la ocasión de aumentar productividad, generar ingresos y captar nuevos clientes. Las dimensiones de la transformación digital son sesgos de esta madurez, los cuales facilitan la planeación estratégica organizacional alineada a la digitalización de sus procesos, así reflexionamos que la dimensión Cambio Organizacional permite que las organizaciones identifiquen la necesidad de nuevas prácticas en el desarrollo de las labores, con objetivos y acciones concretas a elevar esta dimensión. En otro sesgo o dimensión: la tecnología, confirma la urgencia de incorporar herramientas digitales en los procesos de las empresas de hospedaje; ya que el promedio alcanzado queda en el $30 \%$ de los posibles puntos del índice en esta dimensión; en la dimensión de aprendizaje organizacional encontramos el mejor nivel de las 4 dimensiones con un $43.47 \%$ de promedio, pero aún como el resto de las dimensiones sin alcanzar siquiera el punto medio o $50 \%$.

Recursos Humanos resulta ser la dimensión más pobremente evaluada con un promedio de $2.66 \%$, siendo esta dimensión de las primeras que se deben consolidar cuando se habla de Transformación Digital ya que juegan un papel clave en la transformación de toda la cadena de valor de las empresas, pero sobre todo para la gestión del cambio, puesto que son las personas en las que recae el éxito o fracaso de los cambios organizacionales necesarios que apalancan la transformación digital. El estudio evalúa el índice de madurez digital de las empresas y las ubica como lo demuestran los resultados, en niveles incipientes de uso de tecnologías. Con los datos se confirma la urgente necesidad de contar con un modelo de Transformación digital que sea la hoja de ruta de estas organizaciones para romper la inercia de lo conocido y explorar nuevas maneras de ser y hacer las cosas.
Para trabajos futuros sería importante extender esta práctica de medición de las diversas dimensiones organizacionales enlazadas en la Transformación digital, para con ello seguir robusteciendo las hojas de ruta, principalmente de las PYMES, de tal forma que con ello se incrementen las posibilidades de una transformación exitosa. Una vez implementadas estas hojas de ruta o modelos de transformación; también deberán ser medidos, y con ello fundamentar mejores decisiones y acciones por parte de los tomadores de decisiones.

\section{Referencias Bibliográficas}

Alfonso Alfonso, R., Ulacia Oviedo, Z., \& Suárez Mella, R. P. (2019). El Tratamiento de las TICs en hoteles tres y cuatro estrellas de Cuba y Ecuador. Revista San Gregorio,35 (4) 1-15. https://doi.org/10.36097/rsan.v1i35.871

Álvarez Marcos, J., Capelo Hernández, M., \& Álverez Ortiz, J. I. (2019). La madurez digital de la prensa española: estudio de caso. Revista Latina de Comunicación Social, 499 - 520. https://doi.org/10.4185/RLCS-2019-1342

Arnaiz Ramos, F., \& Pinto Valero, S. (2018). Transformación digital en las empresas. 1era. Edición. Madrid: FC Editorial

Barco, J. A. (2016). La Transformación digital: la palanca para una cadena de suministro adaptable, ágil, flexible. Boletín de estudios económicos, 483 - 505.

Becerril Velasco, C. I. (2020). Apropiación de las tecnologías de la información y la comunicación (TIC) para reducir la pobreza en Aguascalientes, México. Entreciencias: Diálogos en la Sociedad con el conocimeinto, 53 - 67. https://doi.org/10.22201/enesl.20078064e.2019.21.69387

Belausteguigoitia Rius, J. C. (2017). Empresas familiares. Su dinámica equilibrio y consolidación. $4^{\text {a }}$ Edición. CDMX: Mc Graw Hill.

Buenrostro Mercado, H. E., \& Hernández Eguiarte, M. d. (2019). La incorporación de las TIC en las empresas. Factores de la brecha digital en las Mipymes de Aguascalientes. Economía, Teoria y práctica., 101-124. https://doi. org/10.24275/ETYPUAM/NE/502019/Buenrostro

Castro, A., \& Gómez Durán, M. (2015). Nivel de Preparación de los Hoteles 4 y 5 estrellas de Cumaná, estado Sucre, Venezuela, para participar en la economía digital. SABER. Revista Multidisciplinaria del Consejo de Investigación de la Universidad de Oriente., 481 - 488.

Cuenca-Fontbona, J., Matilla, K., \& Compte-Pujol, M. 
(2020). Transformación digital de los departamentos de relaciones públicas y comunicación de una muestra de empresas españolas. Revista De Comunicación, 19(1), 75-92. https://doi.org/10.26441/RC19.1-2020-A5

Curbelo, J. L. (2017). Competir en la transgormación digital. Economia Industrial, (404) 135 - 145. Recuperado de https://www.mincotur.gob.es/Publicaciones/Publicacionesperiodicas/EconomiaIndustrial/RevistaEconomiaIndustrial/404/JOS\%C3\%89\%20LUIS\%20CURBELO.pdf

Cruz Estrada, I., \& Miranda Zavala, A. M. (2019). La adopción de las tic en restaurantes de Puerto Nuevo, Rosarito, Baja California. Innovar, 29 (72), 59-76. https://doi. org/10.15446/innovar.v29n72.77932

Cruz Estrada, I., Miranda Zavala, A. M., \& Lobo Rodriguez, M. O. (2019). Innovación mediante las TIC: Retos y oportunidades en las empresas turísticas de Puerto Nuevo, Baja California. El Periplo Sustentable, (36), 372 - 401. https://doi.org/10.36677/elperiplo.v0i36.9142

González Gutiérrez , J. P. (2020). Transformación Digital desde un enfoque normativo.

Gestión y Tendencias GESTEN, 10, 12. https://doi. org/10.11565/gesten.v4i2.90

Grande, M., Cañon, R. \& Cantón, I. (2016) Tecnologías de la información y la comunicación: evolución del concepto y características. Internacional Journal of Education Research and Innovation, 6, 218-230. Recuperado de https://www. upo.es/revistas/index.php/IJERI/article/ view/1703/1559.

Gutiérrez Alva, E., \& Santamaria Mendoza, E. (2018). Problemas de las micro, pequeñas y medianas empresas mexicanas para ser competitivas 2016. Global Conference on business and finance proceedings , 217 - 227.

Hadhri, W., Arvanitis, R., \& Henni Hatem, M. (2016). Determinants of Innovation activities in small and open economics: The Lebanese Business Sector. Journal of Innovation Economics \& Management, 3(21), 77 - 107. https:// doi.org/10.3917/jie.021.0077

Hernández Sampieri, R., Fernández Collado, C., \& Baptista Lucio, P. (2014). Metodología de la Investigación. 6 ${ }^{a}$ Edición. CDMX: Mc Graw Hill.

Herrera Martinez, M. A. (2015). Justicia en la sucesión de empresas familiares. CDMX: Ediciones y gráficos Eón.

Jones, C., Motta, J., \& Alderete, M. V. (2016). Gestión estratégica de tecnologías de información y comunicación y adopción del comercio electrónico en Mipymes de Córdoba, Argentina. Estudios Gerenciales, 4-13. https://doi. org/10.1016/j.estger.2015.12.003

Juca Maldonado, F., Brito, B., García Saltos, M. B., \& Burgo Bencomo, O. B. (2019). La trasformación digital en los procesos académicos de la Universidad como alternativa a la reducción de impacto al medio ambiente. Revista Conrado, 15(67), 309-316. Recuperado de http://conrado.ucf. edu.cu/index.php/conrado

Lombardero , L. (2016). Trabajar en la era digital: Tecnología y competencias para la transformación digital. 1era Edición. Madrid: Lid editorial.

Lopez I Seuba, M. (2019). Internet de las cosas. La transformación digital de la sociedad. 1era. Edición.Madrid: Ra - Ma.

López Jiménez, J., \& Alonso García, M. N. (2019). Los Retos de la igualdad en un escenario de transformación digital. Madrid: Dykinson.

Lorenzo Ochoa, O. (2016). Modelos de madurez digital: ¿En qué consisten y qué podemos aprender de ellos? Boletín de estudios económicos, 573 - 590. Recuperado en: https://www.researchgate.net/publication/313798566_ Modelos_de_Madurez_Digital_en_que_consisten_y_ que_podemos_aprender_de_ellos

Manzano Ibarra, M., Zamora Sánchez, R. y Medina Chicaiza, P. (2019). Propuesta metodológica para la generación de indicadores clave de desempeño apoyada en tecnología de información. 3C Tecnología. Glosas de innovación aplicadas a la pyme, $8(1), 10-29$. https://doi.org/10.17993/3ctecno/2019.v8n1e29/10-29

Martínez Aguiló, J. (2019). Industria 4.0: la transformación digital en la industria. 1era. Edición. Barcelona: UOC.

Mendivil Escalante, V. M. (2015). Metodología para institucionalizar a la empresa familiar y a la empresa mediana. CDMX: Instituto Mexicano de Contadores Públicos.

Monge Malo, L. (2019). Crisis Digital. Por qué las empresas fracasan en su transformación digital y cómo evitarlo. Madrid: Marcombo.

Morales Rubiano, M. E., Ortiz Riaga, C., Duque Orozco, Y. V., \& Plata Pacheco, P. A. (2017). Fuentes de conocimiento e imágenes de la innovación en micro y pequeñas. Revista de investigación desarrollo e innovación., 217-230. https:// doi.org/10.19053/20278306.v7.n2.2017.6081

Muñoz Moreno, J. L., \& Martínez Marín, J. (2018). Aprender en las organizaciones de la era digital: alternativas desde la formacion y hasta la transformación. Cataluña: UOC. Muñoz Sastré, D. (2019). La Cultura Corporativa: claves de la palanca para la verdadera Transformación digital. Prisma Social, 25, 440 - 463.

Ovelar Beltrán, R., Benito Gómez, M., \& Romo Uriarte, J. (2009). Nativos digitales y aprendizaje una aproximación a la evolución de este concepto. ICONO 14. Revista de comunicación y nuevas tecnologías., 31 - 53. https://doi. org/10.7195/ri14.v7i1.332 
Palacios, P. D., Saavedra, M. L., \& Camarena, M. E. (2018). Competencias, talentos y habilidades claves en la PYME manufacturera mexicana. Economía Conyuntural, Revista de temas de coyuntura y perspectivas, 3 (2), 57 - 98. Recuperado de https://www.iies.uagrm.edu.bo/wp-content/ uploads/2019/04/Vol.3-Nro.2-Abr-Jun-2018-3.-COMPETENCIAS-TALENTOS-Y-HABILIDADES-CLAVES.pdf Perez Villamizar, J., \& Mejía Rojas, M. (2018). Análisis del impacto del nivel de transformación digital en la ventaja competitiva de las pymes en Colombia (Tesis Doctoral) Universidad EAFIT repositorio Institucional. Obtenido de https://repository.eafit.edu.co/: https://repository.eafit.edu. co/handle/10784/12591

Puentes Figueroa, C. E., \& Maestre Góngora, G. (2019). Plan estratégico basado en ITIL para mipymes en el departamento de Arauca-Colombia. Lámpsakos,22, 68 - 84. https://doi.org/10.21501/21454086.3280

Ricaurte Quijano, P. (2018). Jóvenes y cultura digital abordajes críticos desde América Latina. Chasqui: Revista Latinoamericana de Comunicación,137, 15 - 30. Disponible en https://dialnet.unirioja.es/servlet/articulo?codigo $=6578582$

Rivera Pesquera, M., \& Mendez Montero, E. (2017). Re evolución digital: Lidera el futuro digital de tu empresa... antes de que desaparezca. Barcelona: Conecta.

Rodriguez Clariana, S. (2018). Empresas Familiares: ¿por que no llegan a la tercera generación? Madrid: ESIC.

Romero Nieva, J., \& Romero Martin, J. M. (2019). Lidera tú empresa en la cuarta revolución ¡Sácale provecho a la transformación digital! Málaga: ExLibric.

Sampietro, S. (2020). Transformación digital de la Industria 4.0. Polo del conocimiento, $1344 \quad-1356$. http://doi.org/10.23857/pc.v5i8.1666

Schallmo, D., \& Williams, C. A. (2017). Digital transformation of business models - best practice, enablers, and roadmap. International Journal of Innovation Management, 1 - 17. https://doi.org/10.1142/S136391961740014X Schumacher, A., Erol, S., \& Sihn, W. (2016). A maturity model for assessing Industry 4.0 readiness and maturity of manufacturing enterpises. Procedia CIRP(52), 161 - 166. https://doi.org/10.1016/j.procir.2016.07.040

Storchevi, M. (2015). The Theory of the Firm and Strategic. Problems of Economic Transitions., 1 - 19. https://doi.org/ 10.1080/10611991.2014.1088357

Urciaga-García, J. I., León-Castro, E., \& Carpio-Mendoza, J. (2019). Los determinantes de la innovaco\}ión organizacional en las empresas PYMES de Guanajuato, México. Global Conference on Business and Finance Proceedings, 499 - 509.
Uribe-Zapata, A. (2019). Cultura digital, juventud y prácticas ciudadanas emergentes en Medellín, Colombia. RLCSNJ, 17(2), 1-19. https://doi.org/10.11600/1692715x.17218 Vainrub, R. (2009). Una guía para emprendedores. Convertir sueños en realidad. México: Pearson.

Valdez Juárez, Luis Enrique, \& García Pérez de Lema, Domingo, \& Maldonado Guzmán, Gonzalo (2017). TIC y la gestión del conocimiento como elementos determinantes del crecimiento de la PyME. Investigación y Ciencia, 25(70),50-62.[fecha de Consulta 30 de Octubre de 2020]. ISSN: 1665-4412. Recuperado de https://www.redalyc. org/articulo.oa?id=674/67451351007

Velázquez Castro, J. A., \& Vargas Martínez, E. E. (2015). De la innovación a la ecoinnovación. Gestión de servicios en empresas hoteleras. Revista Venezolana de Gerencia, 20, 268 - 281. https://doi.org/10.31876/revista.v20i70.19997

Zapata R., G., \& Mirabal M., A. (2018). Capacidades dinámicas de la organización: Revisión de la Literatura y un Modelo Propuesto. Investigación Administrativa, 40,1 - 22. Recuperado de http://www.redalyc.org/articulo. oa?id=456054552003

Zubillaga Rego, A., Aramburo Goya, N., Lorenzo Ochoa, O., North, K., \& Peletier, E. C. (2019). Madurez Digital de la PYME Vasca. Donostia - San Sebastian: Instituto Vasco de Competitividad. Fundación Deusto. 
1.- Nombre del hotel:

\section{2.- Domicilio:}

3.- Tipo de alojamiento:
Hotel
Albergue
Pensión
Casa de huéspedes
apartamento
Otros

4.- Número de habitaciones totales

5.- Tipo de habitaciones

Sencilla

Dobles

Triples

Cuádruple

6.- El hotel cuenta con:
$\neg$ Restaurant
Bar
Lobby
Otros:

7.- ¿Cuántos años lleva funcionando su empresa? años

8.- ¿Cuántas remodelaciones ha realizado?

\section{APRENDIZAJE ORGANIZACIONAL}

9.- Indique cuál de los siguientes métodos utiliza su empresa para definir la tarifa.

Costo $\square$ Oferta/demanda $\square$ Otros ¿Cuál?

10.- ¿Cómo considera que se encuentran sus precios en relación a la competencia?

Arriba de la competencia $\square$ Igual a la competencia $\square$ Por debajo de la competencia $\square$ Lo desconozco

11.- ¿Cuál ha sido su porcentaje de ocupación anual en los años que se indican y a que tarifa promedio?

\section{Indicadores}

$\%$ de Ocupación

Tarifa promedio

Segmento de mercado
2013

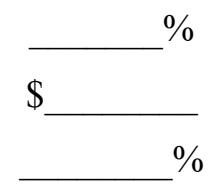

2014

$\%$

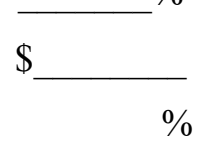

2015

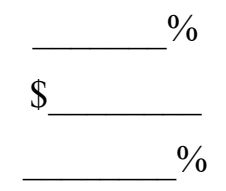


12.- ¿En función a su ocupación que porcentajes de sus ventas provienen de cada uno de los siguientes mercados?

Mercado 2015

Local/Regional

Estatal

Nacional

Internacional

TOTAL

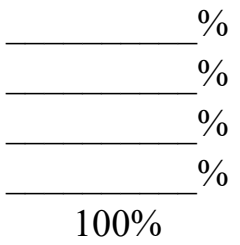

13.- ¿Cuáles considera usted que son los competidores más cercanos al hotel?

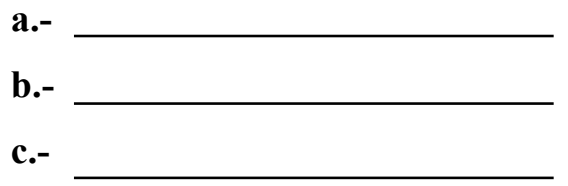

14.- ¿Qué criterios utiliza para identificar a su competencia?

Servicios

Ubicación geográfica

Por tarifa

Ninguna

15.-Qué tipo de estudios realiza la empresa para identificar el rumbo que están tomando sus competidores en relación a la creación de nuevos servicios?

Estudio de mercados $\quad \square$ Análisis FODA $\quad \square$ Market Share $\quad \square$ Ninguna $\quad \square$ Otra ¿Cuál?

16.- A continuación indique cuales son los efectos de los siguientes factores externos:

\begin{tabular}{lcccc} 
Altamente & $\begin{array}{c}\text { Medianamente } \\
\text { Beneficioso }\end{array}$ & $\begin{array}{c}\text { Escasamente } \\
\text { Beneficioso }\end{array}$ & $\begin{array}{c}\text { Sin } \\
\text { Beneficios }\end{array}$ & $\begin{array}{c}\text { Observacio } \\
\text { nes }\end{array}$ \\
Reforma Fiscal & $\square$ & $\square$ & $\square$ & - \\
Impuesto a la nómina & $\square$ & $\square$ & $\square$ & $\square$ \\
Reforma Laboral & $\square$ & $\square$ & $\square$ & $\square$ \\
Reforma Energética & $\square$ & $\square$ & $\square$ & - \\
\hline
\end{tabular}

17.- Indique en que programas públicos se ha visto beneficiado su hotel

Distintivo H $\quad \square$ Distintivo M $\quad \square$ Punto limpio $\quad \square$ Empresas Verdes $\quad \square$ Otros ¿Cuáles? 


\section{8.- Realiza los procedimientos para:}

\begin{tabular}{|c|c|c|}
\hline Ahorrar el consumo energético. & $\mathrm{Si}$ & $\square$ No \\
\hline La reducción del consumo de agua. & $\square \mathrm{Si}$ & $\square$ No \\
\hline $\begin{array}{l}\text { a compra responsable de productos que se } \\
\text { ayan fabricado siguiendo unos estándares que } \\
\text { aranticen en mínimo impacto en el medio } \\
\text { mbiente o el entorno. }\end{array}$ & $\square \mathrm{Si}$ & $\square$ No \\
\hline reciclaje de residuos, materiales y orgánicos & $\mathrm{Si}$ & No \\
\hline
\end{tabular}

\section{RECURSOS HUMANOS}

\section{9.- La empresa cuenta con programas de capacitación.}

De inducción a la empresa $\square$ Por áreas $\square$ De Planeación $\square$ Ninguno

Otros ¿Cuáles?

20.- ¿Con qué periodicidad se capacita al personal?

$\neg$ Seis meses $\square$ Cada año $\square$ No han recibido capacitación

21.-¿Tiene diseñado algún programa de promoción/ascenso para su personal?

$\square \mathrm{Si} \quad \square$ No

En qué consiste:

22.- ¿A través de qué estrategia la empresa reconoce y premia el trabajo de sus colaboradores?

$\checkmark$ Días adicionales (Extras al día de descanso) $\quad \square$ Monetariamente (Horas extras)

Plan de vida y carrera $\square$ Ninguna $\square$ Otras ¿Cuáles?

\section{CAMBIO ORGANIZACIONAL}

23.- ¿En cada periodo presupuestal en qué áreas o departamentos efectúa cambios?

Procesos de gestión administrativa $\square$ Atención al cliente $\square$ Automatización de la infraestructura $\neg$ Ninguna $\square$ Otros ¿Cuáles?

24.- ¿Con la información que se genera actualmente en sus diferentes procesos le permite una adecuada toma de decisiones? (Puede seleccionar más de uno).

Información contable y financiera

De mercado (caracterización del mercado, satisfacción, infraestructura)

Operación y servicios

$\neg$ Infraestructura

$\checkmark$ Administrativo (mantenimiento)

$\square$ Administración (almacén, compras, R:H, costos, auditoría interna de ingresos y egresos)

Operativa (AyB, ventas, Div. Cuartos) 
25.- ¿En cuál de las siguientes estrategias de negocios tiene pensado aplicar cambios en los próximos dos años? (Puede seleccionar más de uno)

$\square$ Adquisición de franquicias $\square$ Alianzas $\square$ Plan de mercados $\square$ Ninguno $\square$ Otros ¿Cuáles?

26.- ¿Indique en cuál de los siguientes procesos se encuentran automatizados y/o tiene pensado invertir en ello en los próximos dos años? (Puede seleccionar más de uno)

$\square$ Reservación $\square$ Check in/out $\square$ Centro de consumo $\square$ Facturación $\square$ Post venta $\square$ Encuesta de satisfacción

$\square$ Ninguno $\square$ Otro ¿Cuál?

\section{TECNOLOGÍAS DE INFORMACIÓN}

Responde a las siguientes cuestiones en relación con su empresa

27.- Enumera en orden de importancia el uso de los servicios de Internet (1) (+) importante, (7) (-) importante

Facebook Twitter Sitio Web Correo electrónico Portal de servicios Web

Buscadores Otros

28.- ¿Con que propósito usa los servicios antes mencionados? (Puede seleccionar más de uno)

Crear nuevos productos o servicios $\square$ Diferenciar productos o servicios

Mejorar productos o servicios $\square$ Crear alianzas $\square$ Mercadotecnia $\square$ Relación con proveedores y clientes

29.- En relación a las siguientes aplicaciones informáticas señale las que habitualmente se utilizan en su empresa: (Puede seleccionar más de uno)

Procesador de textos (WP, Word, otros) $\square$ Base de datos (Access, SQL, otros)

Hoja de cálculo (Excel, otros) $\quad \square$ Programa de gráficos (Publisher, otros)

Contabilidad $\square$ Laboral (Nóminas, otros)

Almacenes $\quad \square$ Facturación

Compras $\square$ Sistema de hospedaje

Otros ¿Cuál?:

30.- Sus aplicaciones actuales logran: (Puede seleccionar más de uno)

Incremento de las ventas $\square$ Incremento de la productividad $\square$ Reducción de costos $\square$ Mejora del servicio a clientes $\square$ Mejora del manejo y administración de recursos económicos

31.- ¿En qué medida su toma de decisiones es apoyada por las TIC o por las aplicaciones seleccionadas anteriormente?
Apoyo total
Apoyo medio
Apoyo escaso
Apoyo nulo 
32.-Conteste las siguientes cuestiones referentes a la actualización de las plataformas con las que cuenta su empresa:

\begin{tabular}{|l|c|c|c|c|c|c|}
\hline & Nunca & $\begin{array}{c}\text { Menos de } \\
6 \text { meses }\end{array}$ & $\begin{array}{c}\text { Entre 6 } \\
\text { meses } \\
\text { un año }\end{array}$ & $\begin{array}{c}\text { Entre 1 a } \\
\text { 2 años }\end{array}$ & $\begin{array}{c}\text { Entre 2 y } \\
\text { 3 años }\end{array}$ & $\begin{array}{c}\text { Más de 3 } \\
\text { años }\end{array}$ \\
\hline $\begin{array}{l}\text { a) ¿Cuándo fue la última vez que } \\
\text { actualizó su equipo de cómputo? }\end{array}$ & $\square$ & $\square$ & $\square$ & $\square$ & $\square$ & $\square$ \\
\hline $\begin{array}{l}\text { b) ¿Cuándo fue la última vez que } \\
\text { actualizó su sistema operativo? }\end{array}$ & $\square$ & $\square$ & $\square$ & $\square$ & $\square$ & $\square$ \\
\hline $\begin{array}{l}\text { c) ¿Cuándo fue la última vez que } \\
\text { actualizó su software de aplicación } \\
\text { administrativa? }\end{array}$ & $\square$ & $\square$ & $\square$ & $\square$ & $\square$ & $\square$ \\
\hline $\begin{array}{l}\text { d) ¿Cuándo fue la última vez que } \\
\text { compró una licencia de software? }\end{array}$ & $\square$ & $\square$ & $\square$ & $\square$ & $\square$ & $\square$ \\
\hline
\end{tabular}

33.-¿Cuál de los siguientes elementos se incluyen en la planeación de TIC's de su empresa? (Puede seleccionar más de uno)

$\neg$ Políticas $\square$ Estándares $\square$ Procedimientos $\square$ Normas $\square$ Ninguna o no tengo

\section{4.- ¿Existe un departamento de informática dentro de la estructura organizacional de} la empresa?

$$
\square \text { Si } \square \text { No }
$$

Si su respuesta es SI.

¿Cuántas personas laboran?

$\square$ Una $\quad \square$ Dos $\quad \square$ Tres $\quad \square$ Cuatro $\quad \square$ Cinco $\quad \square$ Más de
cinco

\section{5.-Enliste las actividades que desempeñan (Puede seleccionar más de uno)}

$\neg$ Desarrollo de Sistemas de Información $\square$ Operación y Mantenimiento de Sistemas

Desarrollo de la infraestructura y Soporte.

\section{¡Gracias por tu colaboración!}

